\title{
Educación y ciencia para salvaguardar el derecho humano al agua: papel de las aguas subterráneas
}

\author{
Emilio Custodio \\ Real Academia de Ciencias. Departamento de Ingeniería Civil y Ambiental, Universidad Politécnica \\ de Cataluña, Campus Nord. C/ Jordi Girona 1-3, Edificio D2(007), 08034 Barcelona. \\ emilio.custodio@upc.edu
}

\begin{abstract}
RESUMEN
Todo ser humano tiene derecho a disponer del agua que necesita para su subsistencia y sanidad razonable, en cantidad, calidad, lugar y tiempo, de acuerdo con las costumbres de su entorno. Es un derecho externo a la propia persona. Si se dispone ya de agua, el derecho no da exclusividad de uso ya que debe ser compartida con los vecinos y la sociedad, a fin de que estos puedan a su vez acceder a su propio derecho. Cuando no se dispone de agua, el derecho es al acceso a la misma. Esto conlleva obligaciones, entre ellas la de contribuir al esfuerzo y coste de puesta a disposición de forma adecuada y de su devolución al medio ambiente en condiciones apropiadas. Además de que el agua es un recurso vital para el ser humano, tiene valor económico como factor de producción y es esencial para el ambiente, en el que se generan funciones y servicios ecológicos que también son esenciales para el ser humano. Existe pues una competencia al mismo tiempo que una complementariedad entre uso del agua y ambiente, modulada por el valor económico y social del agua. Esto conlleva consideraciones éticas y morales, que se apoyan en principios científicos y aplican desarrollos tecnológicos. La ciencia es la base necesaria para el conocimiento que fundamenta la buena gobernanza de los recursos de agua y que está condicionada por los aspectos económicos y sociales y sus implicaciones éticas y morales. Este es el conjunto en que se encuadra el derecho humano al agua y su puesta en práctica, pero con un necesario soporte científico. La buena calidad es un aspecto tan importante o más que la cantidad en cuanto al derecho humano al agua. Las aguas subterráneas juegan un papel clave y frecuentemente decisivo, debido a su ubicuidad, gran almacenamiento en los acuíferos, lentitud de respuesta ante acciones exteriores y complejas relaciones con los otros componentes del ciclo hidrológico.
\end{abstract}

Palabras clave: agua subterránea, derecho humano al agua, medio ambiente, obligaciones.

\section{Education and science to safeguard the human right to water: the role of groundwater}

\begin{abstract}
All human beings have the right to be able to access the water they need for their reasonable subsistence, in quantity, quality, place, and time, and according to what is common in their surroundings. This is a right which is external to the person. When water is available to the person, the right is not in exclusivity, but implies the obligation to share it with other neighbours and society, so they can also exert their right to get water in quantity and quality. When water is not available, the right is to access it. This involves duties, such as contributing to the effort and cost of making water available and its safe disposal in the environment. Besides water being a vital resource, it is an essential part of the environment, which provides important ecological functions and services to mankind. Furthermore, water has an economic value as a factor of production. Thus, there is competition for the use of water and at the same time complementarity between water use and the environment, nuanced by the economic and social value of water. This brings ethical and moral considerations, based on scientific principles and applying to technological developments. Science is the necessary basis for understanding what is needed for sound governance. However, economic and social considerations dominate the scene, with the related ethical and moral conditions. Good water quality is an aspect which is equally or more important than quantity as far as the human right to water is concerned. Groundwater plays a key and often decisive role, due to its ubiquity, large aquifer storage, long associated response to external action and complex relationships with the other components of the hydrological cycle.
\end{abstract}

Keywords: duties, environment, groundwater, human right to water. 


\section{Introducción}

En las recientes décadas se ha ido desarrollando la carta de los derechos del hombre, bajo el liderazgo de Naciones Unidas y de muchas otras organizaciones gubernamentales, de la sociedad civil y religiosas. Entre estas últimas tiene relevancia el cristianismo y en especial la Iglesia Católica (Benedictus XVI, 2009). No son aspectos sencillos, de modo que en su concepción pueden introducirse otras ideas que tienen poco o nada que ver con el objetivo primordial. Esto requiere transparencia para que la propia humanidad, a la luz de los principios éticos y morales, trate de reconducir desviaciones y tergiversaciones.

El derecho de todo ser humano al agua ha merecido atención, con una declaración formal por parte de Naciones Unidas (UN, 2010), en la que las ideas de Gleick (1998) tuvieron una notable influencia. Este derecho al agua potable va muy ligado al del saneamiento, que también se incluye, aunque haya tenido menor desarrollo posterior. En su plasmación mediática se han producido con frecuencia desenfoques, bienintencionados unas veces e interesados otras, con estereotipos que representan situaciones penosas pero extremas y que con frecuencia son atribuibles a otras causas, que no están necesariamente en relación con el agua propiamente dicha, que no se hacen explícitas y que carecen de soporte científico y tecnológico. Por ello, el fomento de foros de discusión abiertos en los que se pueden hacer aportaciones positivas, sin limitaciones doctrinales previas y con la preparación de conclusiones no condicionadas, orientativas pero no vinculantes, es una iniciativa laudable. Tal es el objetivo fundacional de la Pontificia Academia de Ciencias, del Vaticano.

Para tratar del derecho humano al agua tuvo lugar en Ciudad del Vaticano, en los días 23 y 24 de febrero de 2017, dentro de la Cátedra del Diálogo y la Cultura del Encuentro, una reunión amplia de expertos que abarcó un muy amplio temario, desde la ciencia hasta la sociedad y los aspectos intangibles. Se trató del Seminario-Taller: "el derecho humano al agua: aportes y perspectivas interdisciplinarias sobre la centralidad de las políticas públicas en la gestión de los servicios de agua y saneamiento". En el mismo se presentó una primera versión del presente escrito. Se pusieron a disposición muchas de las comunicaciones, algunas de ellas de notable relevancia, pero no se han publicado ni se han hecho públicas. Se prepararon conclusiones (PAC, 2017), pero apenas se dispuso de tiempo de discusión, se dio por bueno el texto preliminar y no se incluyeron observaciones. El diseño del contenido partió parcialmente de un Taller sobre uso de los recursos naturales (WSH, 2014), que asimismo informó una porción del contenido de la Encíclica Laudato Si' (Franciscus I, 2015), con un enfoque más material que espiritual.

\section{Contenido del derecho humano al agua}

Cuando se habla del derecho al agua de los seres humanos se hace una gran simplificación de los aspectos que influyen en su concepto. El agua es un mineral muy especial ya que tiene un contenido económico como factor de producción y como bien deseado y apropiable, pero muy por encima de ellos tiene también un carácter vital, ya que es necesaria para la vida y para la vida humana en particular. Además, aporta dignidad al ser humano y permite sus actividades. Lo primero puede ser objeto de mercado si se dan condiciones de competencia y puede asociarse a un precio, pero lo segundo va más allá del economicismo y entra en el ámbito de los derechos del ser humano. Por esa razón es objeto de especiales consideraciones éticas y morales, ya que no sólo entran juego las costumbres y buen comportamiento según las reglas sociales, sino también valores básicos fundamentados en la Ley $\mathrm{Na}$ tural, impresa por Dios en cada uno de los seres humanos y explicitada en las culturas judeo-cristianas en los Diez Mandamientos.

La declaración de Naciones Unidas (UN, 2010) se refiere al agua potable, aunque en su tratamiento incide también de algún modo en el agua doméstica y la necesaria para actividades primarias en las sociedades más pobres. Incluye el saneamiento, aspecto que aquí no se considera, para centrar la presentación en el derecho al agua. La visión de este derecho al agua que se desarrolla es más amplia que el que se considera en UN (2010), ya que se quiere ir más allá de la mera subsistencia y ver al ser humano en su particular contexto de cada caso. Por esta razón no se dan cifras ni de umbrales ni de estándares ni de realizaciones. Para ello véase Martínez-Santos (2017). El énfasis en las aguas subterráneas es debido a su importancia relativa y en aumento, a que provee una fuente confiable para demandas rurales, semiurbanas y aun urbanas, por sí misma o cada vez más en sistemas integrados de recursos de agua, con consideración de cantidad y calidad. Aproximadamente una tercera parte de la población mundial depende exclusivamente de las aguas subterráneas para su suministro doméstico (Grönwall y Danert, 2020).

Hay derechos que son propios de la condición humana, tales como como los referentes a vivir y expresarse con libertad, al honor y a las creencias. Su ejercicio sólo requiere que otros no la coarten. Su límite es el de que los otros también puedan ejercer sus 
derechos. Esto último es una obligación de cada uno respecto a los otros. El derecho al agua es diferente, pues es externo a la persona, ya que requiere un acceso en competencia o una provisión por terceros. El ejercicio de este derecho conlleva restricciones para no perjudicar los derechos de otros, contemporáneos y también futuros.

Una cosa es el derecho al agua y otra bien distinta es como ejercerlo y cómo los que tienen capacidad de decisión lo materializan en un entorno con limitaciones, incluyendo la cantidad y calidad del agua. A nivel general, aun siendo limitada, con carácter general el agua no es escasa en cantidad, aunque puede serlo localmente y en determinados momentos, en especial por mala gestión y falta de infraestructuras más que por estricta escasez. La escasez se suele manifestar de forma más aguda cuando se considera la calidad, tanto desde el punto de vista de la salinidad como de solutos inconvenientes o nocivos, turbiedad y materia en suspensión o presencia de vectores de enfermedades.

El agua no se consume sino que tras su uso vuelve al ambiente, una parte a la atmósfera como vapor y otra en forma líquida, pero con modificaciones físico-químicas y la adición de substancias disueltas y en suspensión, que pueden degradar las fuentes de recursos de agua y así interferir con la posibilidad de que terceros puedan ejercer su derecho al agua. Como consecuencia, el derecho al agua lleva aparejado también la obligación de que se haga de forma compatible con el buen estado del medio ambiente en el que los seres humanos viven y obtienen alimentos y bienestar, además de respetar intangibles estéticos y religiosos. Los seres humanos a considerar, no sólo son los del entorno, sino otros más alejados a los que les llegarán los efectos de las acciones, unas veces al mismo tiempo o poco después y otras con notable retraso temporal. Esto es especialmente notorio cuando las perturbaciones debidas a la devolución del agua usada al ambiente se propagan a través del terreno y de las aguas subterráneas. El retraso puede ser de años o de muchas décadas, con modificaciones complejas debidas a los procesos físicos, físico-químicos y químicos que se producen en la naturaleza.

La naturaleza es variable en el espacio y en el tiempo. Para que se pueda hacer realidad el derecho humano al agua, se requieren intervenciones humanas basadas en la ciencia y en la técnica. Pero esas medidas suponen acciones, obras, infraestructuras, consumo de energía y esfuerzo humano que no son gratuitos y que deben aportarse de alguna manera por la sociedad. Esto influye en la puesta en práctica del derecho humano al agua y en cómo repartir entre sus destinatarios un recurso que, bajo condicionantes de espacio, lugar, cantidad y calidad, es limitado y pue- de ser escaso para la posible demanda que se pueda generar, pero que raramente lo es para atender al abastecimiento de las necesidades básicas humanas, si existen las infraestructuras mínimas necesarias. Esa escasez en sí misma es matizable y depende mucho de la demanda mínima de agua de un cierto individuo y del grupo social en el que desarrolla su actividad. La escasez tiene un fuerte carácter relativo y apreciativo. Su umbral suele estar muy por encima de las estrictas necesidades vitales.

Todas esas consideraciones llevan a que el ejercicio del derecho humano al agua debe ir acompañado del cumplimiento de obligaciones, tanto de los individuos como de la sociedad. Estas obligaciones incluyen la vigilancia de que el recurso mantenga la cantidad y la calidad, evitar vandalismos, solucionar pleitos por el acceso, etc., y en especial para cubrir de algún modo los costes de poner el agua a disposición. Esto último se refleja frecuentemente en una tarifa explícita o encubierta, pague quien la pague: el usuario, terceros o la sociedad en general. Esto vale tanto para ambientes ricos como pobres, tanto desde el punto de vista económico, como de conocimiento y como de recursos de agua. Los que tienen mayor capacidad de acceder a recursos de agua tienen el deber moral y ético de auxiliar a aquellos con acceso limitado o que carecen de los mismos, además de aportarles una gestión eficaz. Esto no extingue las obligaciones y deberes de los más pobres, aunque se reduzcan al mero aporte personal. La existencia de tarifas por recibir el derecho al agua está justificada por los esfuerzos humanos y materiales que deben ponerse en práctica para que el derecho se materialice, tanto en calidad y calidad, como en lugar y tiempo. La forma de soporte por parte de los más pobres se puede y se suele concretar en el pago de tarifas diferenciadas sociales o en el aporte de esfuerzo personal para procurarse el agua y/o para el servicio comunitario.

Lo expuesto anteriormente es desde el punto de vista de que tenga acceso al agua el que no la dispone, como derecho humano. También hay que considerar la situación de los humanos y grupos humanos que tienen acceso a abundante cantidad de agua de buena calidad, naturalmente o por obras de captación o por disposiciones administrativo-legales, incluyendo los derechos consuetudinarios o de herencia. En este caso, el derecho al agua es para satisfacer sus necesidades razonables y sin que se perjudique la calidad, de modo que terceros puedan ejercer también el derecho al agua y que la sociedad reciba y redistribuya al menos una parte de los beneficios del aprovechamiento económico de esa agua.

De lo anterior se deduce que el derecho al agua de cada ser humano es una abstracción, cuya materializa- 
ción depende de un entramado social que lo garantice y que en su conjunto cubra los costes de la disponibilidad y de garantía de disponibilidad de la cantidad y calidad del agua necesaria y de su devolución al medio ambiente de forma que no se mermen los derechos de terceros. Todo ello ha de ser compatible con los otros usos del agua que también son esenciales al ser humano, tales como la alimentación, el vestido, la producción de bienes necesarios, el saneamiento y el goce estético del paisaje. Todo esto se plantea en un determinado contexto temporal, pero también de traslado a tiempos futuros, en especial cuando se trata de aguas subterráneas y de la introducción de contaminantes en el ciclo del agua, tanto los puntuales como los difusos. Entre los difusos están los asociados a la agricultura y la ganadería extensiva y también los aerotransportados a partir de emisiones directas o indirectas a la atmósfera.

\section{El agua en la Naturaleza: funciones y servicios ecoló- gicos para el hombre}

El agua juega un papel esencial en la naturaleza, el cual tiene muy variados aspectos, desde los climáticos a los geológicos, pero adquiere especial relevancia en lo referente al ambiente y a los seres vivientes. Esto es objeto de la ecología. La satisfacción de las necesidades de agua vitales y económicas del hombre se hace en buena parte en competencia con la ecología, de cuyo ámbito el hombre también forma parte y recibe servicios. Esta competencia por un recurso limitado, aunque no necesariamente escaso, supone establecer reglas para el reparto y para buscar la complementariedad. Estas reglas han ido cambiando a lo largo del tiempo y son distintas según los ámbitos, pero en cualquier caso suponen gestión y una cierta planificación. Estos aspectos, junto con los económicos, sociales y éticos, se combinan en la buena gobernanza. Todo ello necesita un soporte, que es necesario y que está en relación con la posibilidad de ejercer y satisfacer el derecho humano al agua. Se trata de la ciencia y de los desarrollos tecnológicos consecuentes, además de la disponibilidad ineludible de datos fidedignos y suficientes y de actuaciones transparentes. La competencia entre uso humano y disponibilidad del agua en la naturaleza se plantea realmente en términos más amplios, ya que las funciones ecológicas son importantes para la vida y desarrollo humanos. En especial, la naturaleza provee servicios ecológicos diversos (MEA, 2005), que el hombre ha venido utilizando y utiliza y que pueden ser muy importantes para los humanos más pobres. Unos servicios ecológicos tienen valor económico medible, otros tienen un valor social de más difícil valoración y otros son intangibles, pero todos ellos son necesarios y deben ser tenidos en cuenta al considerar la materialización del derecho humano al agua. De ahí que la plasmación en la práctica de los derechos humanos al agua deba combinarse con los derechos del hombre a los servicios ecológicos, en parte también esenciales, como la provisión de alimento y vestimenta y también de regulación y mejora ambiental. Es algo frecuentemente olvidado. Estos aspectos se presentan a veces como derecho de la naturaleza. Esto se puede admitir como una forma coloquial de decir las cosas, pero estrictamente no existe tal derecho ya que la naturaleza no tiene valor si no es en cuanto a la existencia del hombre que la vive, usa, disfruta y conserva y para el que dicha naturaleza constituye un patrimonio. Así, el real sujeto de derecho no es la naturaleza sino el propio hombre, que es el que, a través de sus modos institucionales representativos, tiene que ejercer la defensa de esos derechos, de forma acorde con sus propios derechos al agua.

\section{El conocimiento científico del agua como soporte básico}

La ciencia ha sido, es y será el soporte básico necesario para lograr eficazmente y sin costosos errores que el agua sea un derecho humano que se pueda materializar, sostenga las funciones y servicios ecológicos, permita el desarrollo económico y social de forma equilibrada y sustentable y permita que las sucesivas generaciones humanas la reciban como patrimonio. Es la forma en que los mandatos morales se traducen en hechos.

La ciencia del agua, vista en su conjunto multifacético, es relativamente moderna. No se asentó con amplitud hasta el siglo XIX, aunque recogía experiencias y conocimientos que vienen desde la antigüedad. La ciencia permitió dos grandes logros en el desarrollo humano, después de su uso ancestral para la producción de alimento con ayuda del regadío, con la consiguiente estabilización de la población en numerosos lugares. Uno de los grandes logros recientes, en realidad doble, se produjo a mediados del siglo XIX en el campo de la sanidad, con la identificación de los gérmenes patógenos y sus aspectos hídricos y a continuación con el saneamiento de los núcleos de población y en especial la desinfección del agua de bebida por cloración. El segundo gran logro corresponde al primer tercio del siglo $\mathrm{XX}$, o a mediados del mismo en países en vías de desarrollo o con mayores disponibilidades de agua, cuando se dispuso de medios para el acceso fácil y económico al agua subterránea. Esto ha permitido que las poblaciones y el medio rural tengan 
acceso a agua exenta de gérmenes patógenos, limpia y generalmente de buena calidad mineral.

La consecuencia de ambos logros ha sido una drástica reducción de la morbilidad y mortalidad de la población por enfermedades de origen hídrico y una muy notable mejora en la supervivencia infantil. Además, en el caso del agua subterránea se ha producido una notable liberación de esfuerzo y tiempo que antes se consumía diariamente para proveer agua a las familias, en especial por mujeres y niños. Esto último ha permitido mejorar la escolarización infantil, la mayor atención a la familia y que se puedan ejecutar otras tareas económicas y culturales que produzcan beneficios familiares y comunitarios.

Los cambios mencionados han estado y están acompañados por un notable aumento de la población y por la modificación de la distribución de la pirámide de edades y del potencial laboral. Hay quien ve en ello una seria perturbación y amenaza, a nivel local y también general, y la fuente de presiones migratorias y sobre la captación y uso de los recursos naturales. Es una visión parcial y sesgada, que puede tener matices maltusianos. En realidad, lo que se está observando es una lenta evolución transitoria global hacia un nuevo paradigma poblacional, todavía objeto de diversas teorías, con los conflictos que acompañan a cualquier evolución. Incluso, en ausencia de otras perturbaciones sobreimpuestas, es posible que no se llegue a una estabilización hasta transcurridas muchas décadas. Todo ello va además muy ligado a otros cambios de paradigma, como son los referentes a la energía, la alimentación, la globalización compatible con las peculiaridades y sentimientos de las comunidades humanas y con un modo de vida que no produzca residuos y que substituya a la actual "cultura del descarte", como enfatiza la doctrina de la Iglesia Católica ya desde décadas anteriores. La toma de posturas ideológicas y dogmáticas durante un cambio es un peligroso ejercicio que es propenso a errores, daños y sufrimiento para el ser humano. El caminar en esas condiciones requiere ciencia y humildad en la interpretación de los resultados y su puesta en perspectiva ante una humanidad esencialmente dinámica, en un ambiente cambiante naturalmente. En la concepción judeo-cristiana son individuos que están en tránsito hacia una vida futura, informada por la Revelación.

El marco de la ciencia del agua es el de procurar la solución de los problemas de la humanidad actuales y futuros previsibles para el cuidado de la "casa común", pero la capacidad de llegar a soluciones reales está en que se considere a esta casa común como un lugar temporal ("vehículo común") en el camino hacia la trascendencia y, por tanto, con viajeros cambiantes y con circunstancias en evolución, a veces rápida. Así, la sustentabilidad adquiere una nueva perspectiva que pasa de ser estacionaria a ser dinámica.

La ciencia no aporta ni puede aportar certezas en el campo de las ciencias de la tierra y de la naturaleza, ya que se trata de procesos que tienen una importante incertidumbre. Esta incertidumbre es en parte intrínseca, pero suele estar dominada por una incertidumbre de conocimiento, tanto de la fenomenología como de los parámetros y datos. No por eso la ciencia deja de tener valor, sino que por el contrario lo tiene mayor, ya que pone de manifiesto las incertidumbres y da modos para analizarlas, estimarlas y considerarlas.

El conocimiento de la incertidumbre permite saber y medir el riesgo genérico y el riesgo específico de las actuaciones y decisiones que deben tomarse y que no se pueden soslayar. No tomar una decisión es ya una decisión, que entraña responsabilidades. Las incertidumbres debidas a deficiencias observacionales y limitación de recursos humanos y materiales pueden reducirse con mejores técnicas y más esfuerzo humano y económico, pero con un límite definido por el punto en que la mejora que permite reducir el coste de errores de gestión ya no compensa la mayor inversión en conocimiento. Una gran dificultad para estas valoraciones es cuantificar la parte que afecta al propio ser humano, lo que entraña complejas consideraciones éticas y morales y donde los principios religiosos tienen un gran peso.

El derecho humano al agua conlleva la obligación de aportar datos fieles, suficientes y con continuidad temporal.

Las incertidumbres temporales son importantes en las aguas superficiales y las espaciales en las aguas subterráneas y en ambas las previsiones a medio y largo plazo. Los compromisos de reparto y uso de los recursos de agua deben tener presente esas incertidumbres y como adaptarse a ellas, de modo que las prioridades éticas, como el derecho humano al agua, tengan asociado un riesgo cuantitativo de fallo en la materialización que sea asumible económica y socialmente. En particular se trata del valor personal que cada cultura atribuye a cada uno en cuanto a daños materiales y corporales, ya que estrictamente la vida tiene un valor infinito pero en realidad limitado por la vida de los otros en condiciones equivalentes y con equidad.

Aún con deficiencias notables y campos abiertos a nuevas necesidades apremiantes de investigación, se puede afirmar, sin pecar de exceso de optimismo ni de vanidad, que el estado de la ciencia del agua y en especial de sus recursos en cuanto a la cantidad está razonablemente avanzado. Ello es cierto a pesar de ser una ciencia joven, incluso en lo referente a las aguas subterráneas, para las que, a efectos prácticos, 
se tiene menos de un siglo de desarrollo y en numerosos aspectos sólo medio siglo. No sucede lo mismo en lo referente a la calidad, donde a partir de una base teórica ya bien asentada, aún queda mucho por hacer, incluso en las limitaciones y umbrales a considerar, aunque ya hay grandes avances en tratamientos, en especial los que favorecen procesos naturales y con membranas, y en capacidad analítica.

\section{El ciclo del agua y los recursos de agua}

El reconocimiento y cuantificación del ciclo hidrológico es el fundamento de la ciencia del agua en la naturaleza a nivel general y de la evaluación de los recursos de agua. Se requiere establecer las íntimas relaciones entre sus componentes. El ciclo hidrológico se mantuvo poco alterado hasta la mitad del siglo XX, cuando la utilización intensiva de las aguas subterráneas introdujo modificaciones importantes (Custodio, 2002; 2010; MASE, 2015) y aún cambios externos que afectan al nivel del mar y muy en especial a las funciones y servicios ecológicos, además de requerir un importante consumo energético. Se ha llegado a proponer la explotación intensiva del agua como un ejemplo paradigmático de la "tragedia de los comunes" (Hardin, 1968). Sin embargo, contra una evolución forzada por los acontecimientos, para la que no se requiere intervención pública (Gisser y Sánchez, 1980), la realidad muestra que es posible un control en sistemas con limitaciones reales mediante dicha gestión pública (Esteban y Albiac, 2011). Todos estos efectos influyen en la materialización del derecho humano al agua. Recientemente se ha adicionado al ciclo del agua la llamada producción industrial de agua, que principalmente es la regeneración y puesta a disposición de agua usada, en su mayoría urbana, con un tratamiento apropiado, y la desalinización de agua marina y del agua subterránea salobre y de la regenerada, todo ello también con un consumo energético importante que los aleja de su utilización como herramientas generales para atender a los derechos humanos al agua. En estos campos ha habido un gran desarrollo científico y tecnológico, que posiblemente aun progresará de forma notable y quizás espectacular en algunos aspectos del tratamiento y de la eliminación de contaminantes.

Siguiendo las definiciones comunes en hidrología, la parte renovable del ciclo del agua se considera como recurso de agua, pero sólo una parte de ese recurso es agua disponible para usos humanos directos. Esta parte incluye la que la sociedad debe destinar para cubrir los derechos humanos al agua. La cuantificación de los recursos disponibles depen- de de muchos considerandos de calidad, económicos, ambientales, sociales, políticos y éticos, de modo que no es el resultado de únicamente considerandos científico-tecnológicos, aunque la ciencia proporciona los métodos de estimación para cuantificarlos, una vez que las condiciones de otra índole han sido fijadas o acordadas (Custodio y Dolz, 2016). Esta es una realidad pocas veces asumida.

Los recursos de agua subterránea van asociados a un volumen de reservas, que en general es mucho mayor que la renovación anual. Esto permite una notable estabilidad frente a las variaciones climáticas y de la demanda de agua, lo que facilita que se pueda cubrir el abastecimiento, necesidades de riego y la atención a los derechos de agua poblacionales sin grandes infraestructuras asociadas. Pero, por otro lado, hace que las perturbaciones que se producen en un momento determinado, por ejemplo por incremento de extracciones o por acceso a un nuevo recurso de agua o por una importante alteración territorial, muestren sus efectos de forma diferida y sin que en las etapas iniciales se tengan efectos fácilmente observables. Esta lenta respuesta produce una falsa sensación de seguridad a los gestores y a la población en general. En realidad se traslada el efecto de las perturbaciones al futuro, con un retraso desde años a muchas décadas, según el tamaño y características de cada sistema acuífero. Esto está fuera de la normal percepción del devenir a escala humana y más allá de la acción política habitual. Tiene consecuencias en la materialización del derecho humano al agua en cantidad y calidad, en especial de las generaciones futuras. La materialización del derecho al agua puede verse afectada si no se toman medidas de conservación o se habilitan nuevas reservas.

La frecuente gran reserva de agua de los sistemas acuíferos frente a la renovación anual permite su consumo progresivo, sea de forma planificada, conocida o inconsciente, para atender a las necesidades humanas, entre ellas el derecho al agua, durante un periodo de tiempo más o menos dilatado. Suele ir acompañado de un aumento progresivo del coste y algunos posibles problemas de calidad. Esta es una realidad en muchas áreas áridas y semiáridas (MASE, 2015). Se trata de una solución temporal a problemas actuales de satisfacción de derechos de agua para obtener un beneficio social que permita abordar un futuro cambio de paradigma. Muchos de los aspectos de sustentabilidad, oportunidad y éticos (Llamas et al., 2009) se han de abordar con los métodos que proporciona el conocimiento científico, si se quiere disminuir el riesgo de fallos, con sus costes asociados. 


\section{La calidad del agua y los derechos humanos al agua}

Buena parte de los problemas de disponibilidad de agua se plantean en áreas áridas y semiáridas y hacen referencia a la cantidad de agua disponible. Pero también hay problemas de agua en áreas húmedas, que afectan al derecho humano al agua, en parte por falta de infraestructuras para hacerla disponible y para paliar el efecto de periodos menos lluviosos, y en parte por problemas de calidad. Los problemas de calidad son también frecuentes en áreas áridas y semiáridas y previsiblemente se incrementarán con el tiempo. Esto puede afectar seriamente a la posibilidad del ejercicio y materialización del derecho humano al agua en esos lugares.

En las aguas superficiales, los problemas de calidad son normalmente de salubridad y de contaminación biológica y por vertidos urbanos e industriales. En muchas áreas rurales pobres de ciertos países, la minería puede ir asociada a vertidos y residuos que pueden crear serios problemas de calidad, que además limitan la materialización de los derechos humanos al agua, salvo que haya buenos controles administrativos, responsabilidad ética de las empresas y de los propios mineros, y buena vigilancia por los habitantes, a los que la sociedad debe apoyar con medios y conocimientos.

Las aguas subterráneas están exentas de gérmenes patógenos, salvo en situaciones especiales de falta de suelo y en acuíferos en materiales gruesos, rocas fracturadas o con desarrollo kárstico. Son bien conocidos los casos de pozos contaminados y de serios problemas en la población que los han usado y los usan. Sin embargo, no es un problema del agua subterránea, sino de construcción de las captaciones, en las que por defectos (falta de sello aislante), mal uso y pobre protección del entorno, las aguas contaminadas superficiales pueden penetrar en la captación por el espacio entre la entubación o revestimiento y el terreno perforado o excavado. Es un problema soluble con buenas prácticas, adecuado mantenimiento y un proceso preliminar de desinfección. Ahí es donde los destinatarios de los derechos al agua tienen el deber de establecer y de obligar a la implantación de buenas prácticas, con el apoyo de la sociedad en cuanto a medios y conocimiento. Esto es particularmente importante en muchas áreas pobres, que se muestran como paradigma de incumplimiento de los derechos humanos al agua, cuando en la realidad se trata de fallos tecnológicos y de implicación de la población en el mantenimiento.

El agua subterránea, convenientemente captada y protegida, es normalmente garantía de agua limpia y sin gérmenes patógenos. No obstante, en ocasiones pueden encontrarse concentraciones altas de componentes disueltos de origen natural que son inconvenientes y/o nocivos por acumulación, cuando su ingesta es habitual. Tal puede ser la presencia de altas concentraciones de iones ferroso y/o manganoso, que son tratables, aunque tiene un coste, o un exceso de flúor y/o de arsénico disueltos, de difícil eliminación de forma económica y sobre cuyo origen, comportamiento y localización la ciencia actual no ha llegado aún a resultados satisfactorios con carácter general y aplicables en lugares pobres.

En otros casos se trata de contaminantes diversos, derivados de la actividad humana, de acceso tanto puntual como distribuido. Es frecuente encontrar altas concentraciones de ion nitrato de origen agrícola, ganadero o del saneamiento local, cuyo origen y comportamiento suele ser conocido, pero con un tratamiento costoso y aparición diferida. Cada vez se tiene mejory mayor información sobre la presencia de plaguicidas aplicados en agricultura, pero se conoce mal sus efectos sobre la salud de los que ingieren habitualmente aguas afectadas. El entendimiento de su persistencia en el agua y movimiento en el suelo y terreno requiere mayores desarrollos científicos. Estas contaminaciones aparecen también en los manantiales por los que descargan los acuíferos afectados y consecuentemente en las aguas superficiales derivadas. Todos estos aspectos son bien conocidos científicamente a nivel básico (GEF, 2013), pero se requieren mejoras prácticas para la materialización del derecho a agua de calidad suficiente, en especial en áreas pobres.

Todos estos aspectos de calidad y en especial algunos de carácter extensivo tienen mucho que ver con el derecho humano al agua, ya que se trata de agua potable o que al menos se pueda potabilizar con medios elementales, seguros y baratos, que estén o puedan estar disponibles en sociedades económicamente pobres y con pocos medios de conocimiento y tecnológicos. Estos aspectos son con frecuencia olvidados cuando se considera preferentemente la cantidad. Sin embargo, pueden ser dominantes y el origen de serios problemas. No siempre la ciencia tiene una solución simple y segura, ya que a veces se trata de procesos complejos, variables de un lugar a otro, de difícil observación y medición, que pueden requerir investigación (lenta y costosa) y cuya corrección, si es posible, requiere inversiones y gastos importantes.

Una situación bien conocida de contaminación natural del agua subterránea, que afecta a sociedades pobres, aún no resuelta satisfactoriamente, es la de una extensa área de Bangladesh y Bengala Occidental (Nickson et al., 1998; Ravenscroft et al., 2013; Fendorf et al., 2010; MacDonald et al., 2016). En ella, las enfermedades hídricas asociadas al consumo dominante 
de agua superficial eran hasta la década de 1980 un gran azote que diezmaba la población, en especial la infantil. La construcción de numerosos pozos para el abastecimiento rural y de pequeños núcleos de población palió drásticamente ese problema al proporcionar agua limpia exenta de gérmenes patógenos. Sin embargo, con posterioridad se ha visto que parte del agua subterránea puesta a disposición contiene concentraciones excesivas de arsénico, que por acumulación ha producido y crea problemas sanitarios serios a una parte de la población, sin que haya alternativa a la vista. El área mejoró sanitariamente de forma espectacular con el acceso al agua subterránea y con ello se honró el derecho de la población al agua de bebida, pero han surgido otros problemas sanitarios, menos agudos que los anteriores, pero que no por ello dejan de requerir solución bajo la perspectiva del derecho humano al agua y del valor de una vida. La ciencia no ha podido aún aportar soluciones abordables con los medios económicos y tecnológicos disponibles en sociedades pobres, aunque ya se ha progresado notablemente.

Problemas de exceso de arsénico o de flúor a gran escala, con distintos matices y circunstancias, aparecen en varios lugares de Argentina, Chile, Paraguay, Uruguay, México, China, Cabo Verde, Azores, Tenerife (Islas Canarias), etc. Además, se producen notables problemas de salinidad natural de origen climático en Cabo Verde, Islas Canarias, Norte de Chile, Norte de África, etc.

Los problemas de calidad suelen estar asociados a las mayores dificultades para hacer efectivo el derecho humano al agua potable y es cuando el cumplimiento de las obligaciones de la población, incluyendo a los más pobres, tiene mayor importancia para poder abordar eficazmente los problemas, al menos los más agudos.

Cada vez se está poniendo mayor atención a la presencia de contaminantes de interés (preocupación) emergente en las aguas de abastecimiento. Estos contaminantes son muy diversos e incluyen fármacos, cosméticos, disruptores endocrinos y drogas de abuso de uso frecuente y también tratamientos del ganado con drogas y antibióticos, que actualmente se usan incluso en áreas pobres. Alguno de esos contaminantes son de difícil tratamiento y degradación. Se conocen mal sus efectos sobre la población a las pequeñas concentraciones a las que se encuentran, pero pueden ser no despreciables por acumulación. Es posible que el paso por el suelo y el terreno y una larga detención en los acuíferos ayude a atenuarlos y destruirlos, pero aún se carece de estudios científicos suficientes. La creación de barreras reactivas parece un camino viable y asequible, pero se está en las etapas inicia- les. Aparecen problemas éticos relacionados, pero en principio no afectan a los derechos humanos al agua más que otros aspectos de calidad, pero se requiere que la población cumpla con sus obligaciones de evitar su presencia y mantener las barreras naturales (como es el suelo) para evitar su difusión y favorecer la eliminación.

\section{Ciencia del agua, sociedad y buena gobernanza}

La ciencia del agua es la base del conocimiento de los recursos de agua y el soporte de la tecnología que la apoya. Pero, siendo necesaria para actuaciones fundamentadas y eficaces, no es más que un aspecto que no está entre los que realmente llaman la atención de la sociedad y medios de comunicación y conforman la materialización del derecho humano al agua. El agua, sus recursos, su uso, su devolución al ambiente una vez usada, los beneficios y costes asociados, su papel en la sociedad y otros varios considerandos van mucho más allá de la ciencia y de la tecnología. Las actuaciones y toma de decisiones, una vez informadas por la ciencia -o sin ella si no están disponibles- se basan en consideraciones económicas y sociales, con implicaciones en campos tan diversos como el empleo, la demanda o generación de energía, la producción y seguridad alimentarias y de provisión de fibras y la balanza de comercio exterior. Las decisiones se toman en último término por los políticos, según sus apreciaciones e intereses y de acuerdo con los compromisos adquiridos o que se quieran potenciar. Aquí los expertos del agua juegan como mucho un papel de asesoría, si son invitados a ello, a menos que exista una legislación que potencie su intervención y la sociedad civil lo demande. La sociedad civil bien estructurada, apoyada en un buen y adecuado soporte científico, es algo necesario para realizar el derecho humano al agua.

Lo descrito anteriormente no es una situación rechazable, sino que refleja la realidad y está de acuerdo con el papel del agua, su valor vital y sentimental y su peso económico. Sin embargo, en áreas desarrolladas e incluso de agricultura intensiva, el real peso económico del agua suele ser menor que el que comúnmente se le atribuye y puede ser mayor el efecto de empleo. Lo que conforma las decisiones pueden y suelen ser otros considerandos. Es fácil que se cometan errores en las actuaciones. Estos son aceptables si su coste social es menor que el coste de evitarlos, aunque dicho coste se aminora si se parte de un buen conocimiento y una buena información abierta a todos los interesados, en especial si se trata de instituciones. Estos costes se entienden que son materiales y no limitaciones a la vida. 
Una importante objeción a una actuación y gestión dominada por condicionantes políticos es la de la larga duración de los efectos asociados, en especial los referentes a la explotación y alteraciones del agua subterránea, que rebasa lo que es común en el devenir político. Por lo tanto, no se valora adecuadamente el futuro, al que se pueden trasladar costes que deberían haber sido asumidos dentro de una determinada legislatura política, además de posibles mermas del patrimonio hídrico, sin que estas pérdidas vayan acompañadas de los correspondientes réditos compensatorios. Eso es especialmente cierto para las grandes obras hidráulicas que se desarrollan a lo largo de varias décadas y para los acuíferos de tamaño mediano y grande en los que la respuesta ante acciones progresa durante décadas.

Las decisiones a medio y largo plazo, es decir de lustros a algunas décadas, han de estar informadas y controladas dentro de una planificación flexible, con apoyo político. Esta planificación y su actualización y re-direccionamiento deberían estar aconsejadas por las instituciones adecuadas de una sociedad civil bien estructurada y representativa, en la que las instituciones académicas tienen un papel importante, pero a compartir.

Todo ello conduce a la necesidad de avanzar hacia una buena gobernanza, de la que es responsable el gobierno y autoridades públicas, que se basa en reglas pactadas entre todos los implicados y que obligan a las partes y en una institución representativa que reúna a la administración, usuarios directos e indirectos del agua y la sociedad civil. Dentro de la buena gobernanza es donde debe tener lugar el reconocimiento e implementación de los derechos humanos del agua, conformados bajo principios éticos aceptados por la sociedad civil. Es importante el apoyo de los principios morales que se derivan de la doctrina y experiencia de las instituciones religiosas, dentro de su libertad propositiva, que debe ser independiente de la de la sociedad civil. Esta buena gobernanza es tanto a nivel de pequeños grupos humanos como de grandes unidades territoriales

La educación de los ciudadanos es un aspecto de gran importancia para la buena gobernanza y para el reconocimiento y aceptación de los derechos humanos del agua, conjuntamente con las obligaciones que conllevan. Los principios científicos son los que deben informar de forma independiente y sin sesgos ideológicos y doctrinales una buena educación en los distintos niveles y ámbitos. Es un campo especializado en el que los profesionales de la ciencia y de la técnica no suelen estar preparados y que corresponde a otros especialistas, pero trabajando coordinada y conjuntamente. Uno de los deberes asociados al reconocimien- to eficaz de los derechos del agua por sus receptores concretos es el de educarse y transmitir la educación, así como vigilar que esta educación no esté sesgada o desvirtuada por sectarismos y oportunismos.

Si los gastos asociados a la puesta a disposición del agua y su vertido final adecuado no se cubren por los beneficiarios y por los que reciben la materialización del derecho al agua, sean estos los individuos o la sociedad en que viven, mediante el oportuno sistema de compensaciones internas, lo que se hace es consumir un recurso material (recurso de agua, infraestructuras, sistemas asociados) que no se repone ni se mejora adecuadamente y que con frecuencia sólo se mantiene en mínimos.

El proceso tiene un punto final en el que se requieren inversiones importantes futuras, que puede que no estén disponibles cuando sean perentorias. De no producirse la actualización, no sólo se degradaría o suprimiría la materialización del derecho al agua, sino que se resentiría seriamente todo el abastecimiento humano. Es algo que está sucediendo actualmente y podría originar una crisis en un futuro no lejano, por no adecuar las tarifas, entre otras causas por cobardía política y por no saber trasladar a la población la realidad del problema. Usando la similitud con la "burbuja inmobiliaria" que tanto daño social ha hecho recientemente y que continúa haciendo en un buen número de países, se puede designar como "burbuja de agua" (Cabrera et al., 2012). En todo ello subyace un problema ético relativo al traslado de costes al futuro, cuyos ciudadanos deberán pagar los costes de beneficios que no han recibido, además de heredar un patrimonio mermado.

\section{Derecho al agua y requerimientos de estudio, infor- mación y observación}

La buena gobernanza del agua y el reconocimiento e implementación de los derechos humanos del agua deben basarse en suficientes estudios, adecuada información y observaciones apropiadas, tanto de las variables hidrológicas como de las económicas y sociales. Una parte de la responsabilidad es gubernamental, que debe ejercerse activa e inalienablemente. Pero otra parte corresponde a los usuarios del agua, que deben costearla y colaborar en las mediciones. Esta obligación se extiende, a su nivel, conocimiento y posibilidades, a los que reciben los beneficios del reconocimiento y puesta en práctica de los derechos humanos al agua.

La buena gobernanza del agua supone poner atención a las condiciones que se puedan derivar del clima futuro, dentro de sus grandes incertidumbres y considerando que lo que resulte pueda ser diferente de lo 
que se vislumbra. El cambio climático es una realidad física bien conocida y documentada en el pasado y que va a continuar, sea cual sea la influencia antrópica que se pueda adicionar. Se requiere una actuación prudente y libre de ideologías preestablecidas y la humildad de reconocer que las herramientas de análisis no sólo son imperfectas, sino que la naturaleza es más poderosa que la acción humana y que tiene una notable resiliencia. La planificación a largo plazo requiere considerar el cambio global, que es menos incierto, más gestionable y que puede tener serias implicaciones en cuanto al respeto del derecho humano al agua, con condiciones más favorables en unos lugares y más difíciles en otros.

Para poder realizar extrapolaciones fundamentadas y preparar escenarios verosímiles, es necesario disponer de estudios periódicamente actualizados y con el detalle adecuado a la importancia del problema que se plantea. Además, hay que mantener redes de observación y alimentar regular y permanentemente las bases de datos. Es importante considerar la posibilidad de que sean los propios usuarios y las instituciones adecuadas de la sociedad civil, con un protocolo estricto y buen filtrado, quienes puedan aportar e introducir la información que se les encomiende y responsabilice o de la que dispongan. Esto se debe asociar en parte a las obligaciones derivadas del ejercicio del derecho humano al agua.

La buena gobernanza presupone tomar decisiones de forma transparente, basadas en información fiel y al alcance de todos. La transparencia y buena elaboración de la información es además la forma de evaluar cómo se materializa el cumplimiento del derecho humano al agua.

Tal como ha sucedido en el pasado y está documentado con datos de la prehistoria y la historia, los recursos de agua pueden aumentar en unos lugares y disminuir en otros, con un cambio de régimen que puede tener efectos mayores que los cambios en las medias y con repercusiones no lineales en la recarga a los acuíferos. El cambio climático, en su interpretación como variabilidad climática, ha afectado a las poblaciones humanas de muchos lugares, en el número de individuos y en sus condiciones de vida, y ha producido desplazamientos de población, con invasiones y cambios culturales en pocos siglos e incluso algunas décadas.

Aunque las actuales y futuras tensiones sean similares, no necesariamente se va a reproducir el pasado, ya que ahora la población es mucho mayor y se dispone de un sólido conocimiento científico y medios técnicos mucho mejores, pero las reacciones humanas masivas son impredecibles y en parte obedecen a otros impulsos.
La tendencia natural humana es a conservar lo que se tiene en cuanto a cultura y medios materiales y a evitar cambios, lo que supone establecer fronteras y controles (Horvat II, 2018). Esto es ético, aunque puede ser complejo respetar los derechos al agua. Pero, por otro lado, no es posible luchar permanentemente para estabilizar en una determinada posición a una naturaleza que no es estática sino dinámica y mucho más poderosa que el ser humano. Así, cabe pensar en que se puedan producir desplazamientos poblacionales a gran escala, en busca de mejores condiciones de vida. Estos desplazamientos requieren ser encauzados en un mundo globalizado, con principios generales de gestión, políticos y éticos, pero cuya eficacia estará en el tratamiento a menor escala de los problemas más próximos al individuo, en una real aceptación y aplicación del principio de subsidiariedad. En el fondo subyace un dilema ético-moral. Por lo tanto, la aplicación del derecho humano al agua será cambiante. Si no se prevén anticipadamente las soluciones, se reproducirán los actuales problemas de desplazados por diversas causas, entre las que está la disponibilidad de agua para producir alimentos. La ciencia puede y debe aportar soluciones, pero debe ser adecuadamente soportada y sus logros traducidos en soluciones prácticas que beneficien a todos y en decisiones políticas con visión de estado y universal. Aquí de nuevo la ciencia juega el papel de soporte necesario, pero no tiene la palabra final.

\section{Ciencia de los recursos del agua y legislación}

El reconocimiento efectivo de los derechos del agua y su materialización y también el ejercicio de los deberes asociados, requiere de una adecuada y suficiente consideración en la legislación, sea esta civil, social, ambiental o específica del agua, además de su reflejo en la planificación del agua, con atribución de recursos y responsabilidades. Sin embargo, la legislación de aguas o relacionada es con cierta frecuencia poco efectiva y a veces utópica cuando no ha sido suficientemente informada por el conocimiento científico (Custodio y Dolz, 2016) y está encorsetada en principios conservacionistas en vez de dinámicos. La ley de aguas y otras relacionadas, así como sus decretos, reglamentos y normas, no son documentos científicos ni deben serlo ya que tienen objetivos y destinatarios de otra índole, pero deben ser realistas y conforme a lo que el conocimiento científico sanciona como comportamiento esperable actual y futuro, dentro del reconocimiento de los derechos del agua en un contexto ético y moral. 


\section{Referencias}

Benedictus XVI. 2009. Caritas in veritate. Encyclical Letter. The Vatican: 1-127.

Cabrera, E., Pardo, M.A., Cabrera, E. Jr. and Arregui, F.J. (2012). Tap water costs and service sustainability: a close relationship. Water Resource Management Journal, 1-15.

Custodio, E. 2002. Aquifer overexploitation, what does it mean? Hydrogeology Journal, 10(2), 254-277.

Custodio, E. 2010. Aspectos éticos de la dominada crisis del. En: M.R. Llamas (ed.), Implicaciones Éticas en Algunos Debates Científicos. Instituto de España, Madrid, 91-119.

Custodio, E. and Dolz, J. 2016. Ciencia, técnica y derecho de aguas con referencia a las aguas subterráneas. En: A. Embid Irujo (ed.), Treinta años de la Ley de Aguas de 1985. Universidad de Zaragoza, Thomson Reuters Proview, 209-242.

Esteban, E. and Albiac, J. 2011. Groundwater and ecosystems damages: questioning the Gisser-Sánchez effect. Ecological Economics, 70(1), 2062-2069.

Fendorf, S., Michael, H.A. and van Geen, A. 2010. Spatial and temporal variations of groundwater arsenic in south and southeast Asia. Science, 328, 1123-1127.

Franciscus I (2015). Laudato si', mi' Signore. Encyclical Letter. The Vatican. GEF 2013. Trends in groundwater pollution: loss of groundwater quality and related services. In: Groundwater Governance: A global Framework for Country Action. Prepared by E. Custodio for IAH-UNESCO. Global Environ. Facility, GEF 10 3726: 1-76. http:// www.groundwatergovernance.org/fileadmin/user_upload/groundwatergovernance/docs/Themat

Gisser, M. and Sánchez, D.A. 1980. Competition versus optimal control in groundwater pumping. Water Resources Research, 16, 638-642.

Gleick, P.H. 1998. The human right to water. Water Policy, 1(5), 487-503.

Grönwall, J. and Danert, K., 2020. Regarding groundwater and drinking water access through a human rights lens: Self-supply as a norm. Water, 12(2), 419; doi:10.3390/ w12020419

Hardin, G. 1968. The tragedy of the commons. Science, 162, 1243-1248.
Horvat II, J. 2018. Why good fences make good neifgbors. The American Society of Tradition, Family and Property: 1-4.

Llamas, M.R., Martínez-Cortina, L. and Mukherji, A. (eds.) 2009. Water ethics.. CRC Press, 1-368.

MacDonald, A.M., Bonsor, H.C., Ahmed, K.W., et al. 2016. Groundwater quality and depletion in the Indo-Gangetic Basin mapped from in situ observations. Nature Geoscience Letters: 29 August 2016, 1-8. DOI: 10.1038/ NGEO2791.

Martínez-Santos, P. 2017. Does $91 \%$ of the world population really have "sustainable access to safe drinking water"? International Journal of Water Resources Development, doi 10.1080/07900627.2017.1298517

MASE 2015. Aspectos hidrológicos, ambientales, económicos, sociales y éticos del consumo de reservas de agua subterránea en España: minería del agua subterránea en España. Preparado por E. Custodio para UPC y AQUALOGY-Cetaqua, Barcelona, 1-488. http://hdl.handle. net/2117/111272

MEA 2005. Ecosystems and human well-being: wetlands and water synthesis. Millennium Ecosystem Assessment. World Resources Institute, Island Press, Washington, DC, 1-68.

Nickson, R., McArthur, J.C., Burgess, W., Ahmed, K.M., Ravenscroft, P. and Rahman, M. 1998. Arsenic poisoning of Bangladesh groundwater. Nature, 395, 338-.

PAC 2017. Final statement of the workshop on the human right to water. The Pontificial Academy of Sciences. The Vatican. http://www.pas.va/content/accademia/en/ events/2017/water/final_statement.html

Ravenscroft, P., McArthur, J.M. and Hoque, M.A. 2013. Stable groundwater quality in deep aquifers of Southern Bangladesh: the case against sustainable abstraction. Science of the Total Environment, 454-455, 627-638.

UN 2010. The human right to safe drinking water and sanitation. United Nations. General Assembly. Resolution A/ $\mathrm{RES} / 64 / 292$.

WSH 2014. Workshop on sustainable humanity, sustainable nature: our responsibility. Pontificial Academy of Sciences and Pontificial Academy of Social Sciences, 2-6 May 2014. The Vatican.

Recibido: julio 2019

Revisado: noviembre 2019

Aceptado: enero 2020

Publicado: marzo 2021 